\title{
Exterritoriale Ressourcen: Die Diskussion um die Tiefsee, die Pole und das Weltall um 1970
}

\begin{abstract}
The chapter explores how, in the Cold War period, large terrestrial and extraterrestrial regions came into focus as sites of strategic importance, areas of expansion or valuable resources: the deep sea, the polar regions and outer space. While the modern world since the $19^{\text {th }}$ century had been subjected to extensive processes of imperial expansion and territorialisation, these regions long escaped sovereign rule, entering international debate only when technology made national claims viable. The chapter studies political ambitions to set up new political and legal regimes of access. It discusses the contested concept of global commons and its legal equivalent, the Common Heritage of Mankind principle, and argues that seemingly opposing concepts of territorialisation and communalisation were closely related. The commons regimes of the 1970s, it is argued, need to be reinterpreted as a double-edged sword, which failed to introduce a feasible commons-based regime and did not overcome but reinforced the earth's spatial organisation in terms of territory and sovereignty.
\end{abstract}

Im Nordatlantik liegt eine einsame Felseninsel mit dem Namen Rockall. Zuerst tauchte sie in Reisebeschreibungen des späten 17. Jahrhunderts auf, erklommen und kartographisch erfasst jedoch wurde sie erst im 19. und 20. Jahrhundert. Kaum 20 Meter misst Rockall in der Höhe und gerade einmal 30 Meter im Durchmesser. Für Menschen scheint der Felsen ohne jeden Belang zu sein, da er praktisch unbewohnbar ist. Rockall ragt steil aus dem Meer heraus und ist zudem gänzlich karg, dem Wind und dem Wetter ausgesetzt. Dennoch erhielt Rockall während des Kalten Krieges strategische Bedeutung. Im September 1955 gelang es britischen Soldaten, an dem Felsen zu landen und ihn zu erklimmen. Sie zogen den Union Jack auf und brachten eine Gedenktafel zur Inbesitznahme Rockalls im Namen Ihrer Majestät an. Ziel dieser ,letzten territorialen Expansion des Vereinigten Königreichs“1 ${ }^{1}$ war es, gemäß seerechtlicher Tradition und Praxis die Insel und das umliegende internationale Gewässer hoheitlich zu reklamieren. Nach dieser

1 Fraser MacDonald: The Last Outpost of Empire. Rockall and the Cold War, in: Journal of Historical Geography 32 (2006), 627-647, hier 627. Das Vereinigte Königreich erwarb die MGM-5 Corporal aus amerikanischer Produktion im Jahre 1954. Versuche wurden 1959 auf einem Testgelände der Royal Artillery auf South Uist durchgeführt: Fraser MacDonald: Geopolitics and ,the Vision 
Tradition fielen auch die umliegenden Gewässer der Felseninsel unter die nationalen Hoheitsrechte. Rockall lag im Funkbereich des Versuchsgeländes für die Corporal-Rakete, die erste britische Kurzstreckenrakete mit Atomsprengkopf, die auf der schottischen Insel South Uist in den Äußeren Hebriden getestet werden sollte. Der Sowjetunion, dem wichtigsten Kontrahenten im Kalten Krieg, sollte der Aufenthalt zu Spionagezwecken im besagten Gebiet großräumig unmöglich gemacht werden.

In den 1960er Jahren zerschlugen sich die Träume der Briten, eine eigenständige Atommacht zu werden. Das militärische Interesse der Briten am Seegebiet um Rockall hatte sich bald überholt und wurde durch die Aussicht auf ozeanische Ressourcen abgelöst, die internationale wirtschaftliche Interessen auf den Plan brachten. Zum einen galt die Umgebung der Rockall Bank als fruchtbarer Fischgrund. Ebenso wie die Aufenthaltsrechte waren auch die Fangrechte in den Gewässern abhängig von deren Auslegung als nationale Hoheitsgewässer beziehungsweise als freie See. Zum anderen erbrachte der British Geological Survey neue Hinweise auf Öl- und Gasvorkommen im unterliegenden Plateau der kontinentalen Erdkruste. Um seine Souveränität formal zu bekräftigen und die Fangrechte zu sichern, die Rohstofferkundung durch andere Staaten zu untersagen und die Passierrechte militärischer und ziviler Schiffe sowie Flugzeuge im Hoheitsgebiet einzuschränken, inkorporierte Großbritannien mit dem Island of Rockall Act von 1972 den Felsen als Teil Schottlands in sein Territorium. Die Rechtshandlung sollte vor allem die entdeckten Öl- und Gasreserven vor den Ansprüchen Irlands und Islands schützen. ${ }^{2}$

Der britische Regierungsakt war ein einseitiger Sprechakt. International blieb er praktisch folgenlos, denn er fand keinerlei Anerkennung. Eine völkerrechtlich bindende Regelung, die anderen Staaten Vorschriften zum Aufenthalt im Gebiet um Rockall hätte machen können, existierte zu dieser Zeit nicht. Erst die UN Seerechtskonvention von 1982 legte fest:

Felsen, die menschliche Siedlungen oder Ökonomie nicht selbst aufrechterhalten können, sollen weder eine exklusive Wirtschaftszone noch ein Kontinentalschelf besitzen. ${ }^{3}$

Thing'. Regarding Britain and America’s First Nuclear Missile, in: Transactions of the Institute of British Geographers 31 (2006), 53-71.

2 „1972 Island of Rockall Act“, in: legislation.gov.uk. http://www.legislation.gov.uk/ukpga/1972/2 (17. März 2014).

3 Zitat im Original: „Rocks which cannot sustain human habitation or economic life of their own shall have no exclusive economic zone or continental shelf.“, in: United Nations Convention on the Law of the Sea (UNCLOS) 1982, Part VIII, Regime of Islands, Article 121. http://www.un.org/depts/los/convention_agreements/texts/unclos/part8.htm (17. März 2014). 
Der Streit um die Gebietshoheit über Rockall, der bis heute unter den nordatlantischen Schelfanrainerstaaten Island, Irland, Dänemark (für die Färöer Inseln) und Großbritannien ausgetragen wird, ist zu einem Streit um die Definitionshoheit über die Felseninsel geworden. Er dreht sich um die Unterscheidung zwischen Felsen und Insel. Dem Felsen Rockall kommen weder territoriale Gewässer zu, die Fangrechte regeln, noch lassen sich Schürfrechte ableiten. Das Festlandsockelkonzept und die hoheitsrechtliche Zuordnung einer exklusiven Wirtschaftszone (Exclusive Economic Zones, EEZ) von 200 nautischen Meilen gelten nur für solche Gebiete, die als Inseln klassifiziert sind. Inseln wiederum müssen ihre Bewohnbarkeit nachweisen können. Tatsächlich harrte deshalb ein Überlebensexperte und ehemaliger Angehörige des britischen Special Air Service im Jahre 1985 vierzig Tage auf Rockall aus.

\section{Deutungshoheit und Gebietshoheit}

Als Kuriosität ragt Rockall aus einer langen Geschichte der Auseinandersetzung um exterritoriale Gebiete im 20. Jahrhundert heraus - wobei der Begriff exterritorial im Grunde irreführend ist, beruht doch Exterritorialität immer schon auf einer politischen und rechtlichen Vereinbarung. ${ }^{4}$ Zahllose Orte, Personen und Organisationen, vom Diplomaten bis zum Vatikan, gelten in der Folge politischer Vereinbarungen als exterritorial. Sie sind buchstäblich aus der sie umgebenden Hoheitsgewalt ausgenommen und genießen Immunität, doch stehen sie keineswegs außerhalb des Gesetzes. Im Folgenden soll es indes um solche Gebiete gehen, die trotz des beinahe vollständigen territorialen Zuschnitts der Welt bis in das 20. Jahrhundert hinein außerhalb jeglicher Jurisdiktion standen und die erst während des Kalten Krieges als strategische Orte, als mögliche Expansionsräume und Rohstoffspeicher in das Fadenkreuz internationaler und auch globaler Verhandlungen gerieten: die Tiefsee, die Polargebiete und das Weltall.

Rockall markiert gleichsam die Spitze des Eisbergs der Schwierigkeiten, solche Gebiete territorial eindeutig $\mathrm{zu}$ fassen und in verbindlicher internationaler Gesetzgebung zu kodifizieren, sobald mehrere Konfliktparteien involviert sind. Der Anspruch auf solche Gebiete wird notwendig performativ erhoben, denn er kann nicht ontologisch abgesichert, sondern muss vorgetragen beziehungsweise

4 Exterritoriale Gebiete sind Gebiete, die aus politischen Vereinbarungen als keinem nationalen Territorium zugehörig hervorgegangen sind. Der Begriff „exterritorial“ ist kein Quellenbegriff der (UN-) Vertragswerke um 1970, denn in den Verhandlungen ging es um terra nullius, um nicht zugewiesenes und teilweise noch nicht reklamiertes Land. Siehe hierzu noch unten. 
inszeniert werden. Traditionelle Weisen der performativen Vereinnahmung sind die Okkupation und permanente Präsenz sowie die Reklamation aufgrund geographischer Nähe. Zunehmend werden Besitzansprüche auch wissenschaftlich erhoben. Mit Blick auf die Fang- und Schürfrechte in der Region suchten Rockalls Anrainerstaaten Island, Irland, Dänemark und Großbritannien die Zugehörigkeit der Insel zu ihrem Territorium tektonisch zu legitimieren und legten dazu der UN-Kommission für Kontinentalschelfgrenzen (CLCS) ihre Messungen über die Ausdehnung ihrer Schelfe vor. Doch sind auch wissenschaftliche Vermessungsprogramme weder politisch neutral noch objektiv. Sie agieren in staatlichem Auftrag und ihre geomorphologischen Grundlagen sind umstritten. Ein ozeanisches Gebiet lässt sich einem Nationalstaat nicht strikt geologisch, sondern nur im Rahmen internationaler wissenschaftlicher Auslegungen und politischer Abkommen zuordnen. Umgekehrt zeigt Rockall, dass legitimierte Institutionen wie die Vereinten Nationen zwar notwendig, aber kaum hinreichend sind, um über konfligierende Gebietsansprüche und Nachweise zu entscheiden. ${ }^{5}$

Nicht zuletzt illustriert Rockall als Felsen wie als Insel die Beobachtung, dass ein Gebiet erst im Zuge seiner Diskursivierung in der politischen und juridischen Rede und durch performative Praktiken der Beanspruchung und der Anerkennung Gestalt annimmt. Dort, wo die tradierten Wege der Landnahme schwer fallen oder scheitern, werden andere Weisen der sprachlichen und praktischen Einordnung wirksam. Im Jahre 1997 invadierten Aktivisten der Umweltschutzorganisation Greenpeace den Felsen und riefen Rockall zum globalen Gemeingut (global common) aus. Ihr Ziel war es, die von Briten, Dänen sowie Isländern angestrebte Ölförderung im unterseeischen Plateau zu verhindern. Die Erschließung von Ölvorkommen in atlantischen Gebieten wie dem Rockall-Plateau, die wenige Jahrzehnte zuvor als technisch zu aufwendig erachtet wurde, schien inzwischen lukrativ. Unter Berufung auf die oben genannte Kontinentalschelfkonvention der UN beanspruchten die drei Staaten die Ausbeutung für sich, sondierten das Terrain und projektierten Probebohrungen. Die Greenpeace-Aktivisten setzten diesen konkurrierenden Besitzansprüchen ihren eigenen Anspruch entgegen: Sie gründeten auf Rockall den globalen Mikrostaat „Waveland“ und verteilten symbolisch zu erwerbende Bürgerschaftszertifikate über das Internet. Sie brachten ein kleines solarbetriebenes Leuchtfeuer auf der Insel an und versahen es mit

5 Die UN-Kommission für Kontinentalschelfgrenzen (UN Commission on the Limits of the Continental Shelf, CLCS) wurde 1982 in Zusammenhang mit der Seerechtskonvention der Vereinten Nationen (UNCLOS) gegründet. Der Kontinentalschelf oder Festlandsockel bezeichnet den Randbereich des Kontinents, der von Wasser bedeckt ist, aber zur Festlandsmasse gerechnet wird. Die Ausdehnung der Schelfe variiert je nachdem, welche Auslegung und welche wissenschaftlichen Methoden (geologisch, geographisch, ozeanographisch etc.) zugrunde gelegt werden. 
einer Messingtafel, welche die streitenden Ölförderstaaten und die globale Gemeinschaft aufforderte: „Lasset die Sonne und den Wind ihre Arbeit tun. Belasst das Öl unter den Wellen.“6

In diesem Beitrag werden die Vereindeutigungs- und Verrechtlichungsprobleme diskutiert, denen die Meere, die Pole und das Weltall seit der Mitte des 20. Jahrhunderts ausgesetzt wurden. Das Augenmerk liegt dabei auf der politischen Nähe vermeintlich opponierender Strategien der Territorialisierung und der Kommunalisierung von Räumen und auf der engen Verwandtschaft der jeweiligen Eigentums- und Nutzungsregime. Dem Bestreben der nationalen Einhegung von Gebiet stand seit 1970 ein Diskurs der Vergemeinschaftung gegenüber, der terrestrische und extraterrestrische Gebiete als globale Gemeingüter reklamierte und unter dem Prinzip des Common Heritage of Mankind (CHM) unter internationales Recht stellte. Die rechtliche Auszeichnung eines Gebiets als Common Heritage of Mankind beziehungsweise als Gemeinsames Erbe der Menschheit sollte nationale Ansprüche eindämmen. Zugleich sollte das Prinzip solchen Akteuren, die geographisch, technologisch und politisch weniger privilegiert waren, den Zugang zu Gebieten gewähren, die als natürliche Ressourcen attraktiv wurden. Auf beiden Seiten der Debatte waren jedoch die Akteure vor allem die Nationalstaaten, die im internationalen Völkerrecht als einzig legitime Verhandlungspartner anerkannt und aufgerufen waren. In dieser Situation, so die These des Beitrags, waren die um 1970 entstehenden Commons-Regime wenig geeignet, zwischen territorialen und gemeinschaftlichen Eigentums- und Souveränitätsprinzipien zu vermitteln. Vielmehr wurde die Prinzipienfrage auf der globalen Ebene zugunsten neuer territorialer Strategien aufgelöst.

Die folgenden Abschnitte diskutieren diese Zweischneidigkeit der CommonsRegime. Der erste Abschnitt geht der Nähe von Privatisierung und Vergemeinschaftung in Bezug auf irdische und außerirdische Räume nach. Er untersucht die Prinzipien terra nullius und terra communis als verwandte exterritoriale Vereinbarungen. Der zweite Abschnitt studiert die Nähe von Schutz- und Nutzprinzipien in Commoning-Strategien und diskutiert sie als neue Formen der Sicherung von Teilhabe. Der dritte Abschnitt diskutiert die Uneindeutigkeit von Eigentums- und Nutzungsmodi in verschiedenen debattierten Wirtschaftssystemen der Zeit - privatwirtschaftliche und sozialistische Ökonomien sowie Commons-Ökonomien. In einer Situation der Dominanz des Westens im internationalen Diskurs über die Erde, in dem Commoning-Konzepte hauptsächlich von westlichen Akteuren und nach westlichen Ideen definiert wurden, wurde auch die Ökonomie der Commons

6 Zitat im Original: „Let the sun and wind do their work. Leave the oil beneath the waves.“, in: A History of Waveland in Five Chapters. http://www.waveland.org/history.html (17. März 2014). Siehe auch Öl-Rausch round the Rockall, in: Greenpeace Magazin 4 (1997). 
in Begriffen der Territorialität verhandelt. Der vierte Abschnitt fragt daher, inwieweit Commons-Prinzipien selbst territorial waren und diskutiert die um 1970 formulierte Behauptung eines Übergangs vom Territorialitätsprinzip zur „NeoTerritorialität“.

\section{Privatisierung und Kommunalisierung}

Den modernen Bestrebungen der Nationalisierung beziehungsweise der transnationalen Kollektivierung von terrestrischen und extraterrestrischen Räumen liegen viel ältere Prinzipien der Gebietsdefinition und der daraus abgeleiteten Gebietsansprüche zugrunde: Terra nullius und terra communis entstammen dem Römischen Recht und bezeichnen unterschiedliche Konzeptionen irdischer Räume als Niemandsland beziehungsweise als Gemeingut. Die Frage, welche Bestimmung für ein Gebiet, das als Rohstoffspeicher oder Ergänzungsraum umstritten war, völkerrechtlich akzeptiert wurde, entschied über dessen Zuteilung und Nutzung.

Eines der ältesten Beispiele für eine strittige Gebietsdefinition ist das Prinzip der Freiheit der Meere, mare liberum. Hugo Grotius, einer der Begründer des Souveränitätsprinzips, hatte 1609 im kolonialen Streit um Handelsrouten zwischen den Niederlanden und Portugal bestimmt, die hohe See sei nach dem römischen Konzept des ius naturale oder Naturrechts „,common to all and proper to none“: Was nicht umschlossen und gehandelt werden konnte, so seine Ansicht, das konnte nicht besessen werden, woraus folgte, dass die unsteten Ozeane frei seien. ${ }^{7}$ Der im 19. Jahrhundert völkerrechtlich allgemein anerkannte Grundsatz begrenzte nationale Rechte auf eine Küstenzone von drei Meilen. Diese Distanz entsprach der Reichweite der damaligen Kanonengeschütze, die entsprechend der zeitgenössischen technischen Möglichkeiten die Landesverteidigung sicherten. Rechtlich geregelt wurden außerdem die Hochseefischerei und die Handelsschifffahrt. Meeresboden und Meeresuntergrund spielten weder in wirtschaftlicher noch in militärischer Hinsicht eine Rolle. Wie die Hochsee blieben sie im Sinne Grotius' frei - Räume, die rund 70 Prozent der Erdoberfläche ausmachten.

7 „The sea therefore is in the number of those things which are not in merchandise and trading, that is to say, which cannot be made proper. Whence it followeth, if we speak properly, no part of the sea can be accompted in the territory of any people." Hugo Grotius: The Free Sea (orig. Mare Liberum. De Indis, Kapitel 12. Leiden 1609), hg. von David Armitage. Indianapolis 2004, Kapitel 5: „That the sea or right of sailing on it is not proper to the Portugals by title of possession." Siehe auch Norman Weiß (Hg.): Hugo Grotius. Mare Liberum. Potsdam 2009. 
Das 20. Jahrhundert forderte diese jahrhundertealten Arrangements technologisch heraus. In den 1960er Jahren nahm die unterseeische Erdöl- und Erdgasförderung zu und Manganfunde in der Tiefsee versprachen neue Erzquellen. Angesichts einer rapide wachsenden Weltbevölkerung wuchsen die Hoffnungen auf die Meere als Eiweißspeicher wie als ergänzender Lebensraum. Experimente des Arbeitens am Meeresboden in Unterwasserlaboratorien und neue Techniken der Gewinnung von Plankton und Krill aus dem Meer schürten die Erwartungen. ${ }^{8}$ Das Buch Wem gehört der Ozean? beschrieb im Jahre 1969 die Aspekte des neu entfachten Kampfes um die Weltmeere und insbesondere um die untermeerisch „schlummernden Schätze“9 einer breiten Leserschaft: das lückenhafte Seerecht, die überschäumenden wirtschaftlichen Hoffnungen, die Pläne zur militärischen Nutzung sowie die Kehrseite, die Meere als wachsende Müllhalde. Der Streit um ein neues internationales Seerecht fällt demnach nicht zufällig in diese Zeit. Drei große Konferenzen der Vereinten Nationen berieten 1956, 1960 und nochmals ab 1973 über die Neuregelung der ozeanischen Eigentums- und Nutzungsrechte. An deren Ende stand das im Jahre 1982 beschlossene und 1994 ratifizierte Internationale Seerechtsabkommen (United Nations Convention on the Law of the Sea, UNCLOS). Es definierte Territorialgewässer neu und etablierte das Konzept der exklusiven Wirtschaftszonen für den küstennahen Rohstoffabbau und Fischfang. ${ }^{10}$

Im Jahr der ersten Mondlandung war es der zweifache „Griff des Menschen nach bisher unerreichbaren Höhen und Tiefen“, der einen neuen Abschnitt in der Geschichte einzuläuten schien und die Frage aufwarf:

Soll allein das Recht des Erstgekommenen und des Stärkeren gelten, oder aber sollen Weltraum und Meeresboden zur res communis der Menschheit werden? ${ }^{11}$

Terrestrische und extraterrestrische Gebiete, die Grotius' Argumentation zufolge weder schlicht $\mathrm{zu}$ besetzen noch $\mathrm{zu}$ besitzen waren, wurden in den 1950er und 1960er Jahren wissenschaftlich-technisch durchdrungen und symbolisch annek-

8 Christian Kehrt/Franziska Torma (Hg.): Lebensraum Meer, Themenheft Geschichte und Gesellschaft (in Vorbereitung); Sven Asim Mesinovic: Die Eroberung der Meere. Die Unterwasserlaboratorien Helgoland (BRD) und Tektite (USA) im Umweltdiskurs, 1968-1973. Florenz 2012 (Dissertation EUI); Jacob Darwin Hamblin: Oceanographers and the Cold War. Seattle 2005.

9 Joachim Joesten: Wem gehört der Ozean? Politiker, Wirtschaftler und moderne Piraten greifen nach den Weltmeeren. München 1969, Zitat aus dem Klappentext.

10 „United Nations Convention on the Law of the Sea (UNCLOS) 1982“, in: Ocean and Law of the Seas United Nations. http://www.un.org/Depts/los/convention_agreements/texts/unclos/ unclos_e.pdf (17. März 2014); Edward L. Miles: Global Ocean Politics. The Decision Process at the Third United Nations Conference on the Law of the Sea, 1973-1982. Cambridge/Mass. u.a. 1998.

11 Joesten, Wem gehört der Ozean? (1969), 7. 
tiert. Beispiele einmaliger oder permanenter wissenschaftlicher Präsenz sind die erste Antarktisstation, die 1957 von den USA installiert wurde, das Atom-U-Boot USS Nautilus, das 1958 den Nordpol erreichte, das Tauchboot Trieste, das 1960 den knapp 11.000 Meter tiefen Marianengraben erkundete, und die Apollo 11 Mission, die 1969 die US-Flagge auf den Mond pflanzte. Das space race, das die Vormachtstellung im Kalten Krieg im All zu demonstrieren suchte, war auch ein Wettstreit um terrestrische und extraterrestrische Ressourcen.

Bereits das Internationale Geophysikalische Jahr (IGY) 1957/1958 zeigte, dass die materiellen und auch immateriellen Eigenschaften der Erde und des Weltraums von handfester ökonomischer Bedeutung waren. Die geostationäre Umlaufbahn etwa, ein schmales erdnahes Band in einer Höhe von 36.000 Kilometern über dem Äquator, erwies sich als unabdingbar zur Verankerung der Satelliten zur Fernerkundung und Kommunikation zu wissenschaftlichen oder militärischen Zwecken. 1976 entwarfen acht Äquatorialstaaten mit der Bogota Deklaration einen eigenen Eigentumsplan, der den geostationären Orbit als der Erde zugehörige „natürliche Ressource“ deklarierte, die dem Territorium der Äquatorialstaaten zugesprochen und ihrer nationalen Souveränität unterstellt wurde. Ausgesprochenes Ziel des Planes war es, den finanziell und technologisch benachteiligten Äquatorialstaaten Brasilien, Ecuador, Indonesien, Kenia, Kolumbien, Kongo, Uganda und Zaire den gerechten Zugang zu dieser Ressource zu ermöglichen. Diejenigen Teile der geostationären Umlaufbahn hingegen, die über den offenen Ozeanen lagen, sollten als Gemeingut erachtet und deren Nutzung international „zum Wohle der Menschheit“ reguliert werden. ${ }^{12}$ Auch dieser Plan blieb jedoch, entsprechend dem Fall Rockalls, international ohne Anerkennung und praktisch ohne Wirkung.

Jeweils unterschiedliche Definitionen der anvisierten Gebiete legten unterschiedliche Aneignungs- und Bewirtschaftungsweisen nahe. Die Territorialisierung von terra nullius oder Niemandsland stand in der Tradition der nationalen Herrschaftsbildung durch Akte der räumlichen Einhegung und Kolonisierung. Nach Römischem Recht war ein solches Gebiet herrenlos, es unterlag keinem Eigentumsregime. Es war weder besiedelt noch bewirtschaftet und stand daher zur Okkupation frei. In vielerlei Hinsicht schrieb die Vorstellung der Ozeane,

12 Declaration of the First Meeting of Equatorial Countries, angenommen am 3. Dezember 1976 von den Staaten Brasilien, Ecuador, Indonesien, Kenia, Kolumbien, Kongo, Uganda und Zaire. Die Zitate „natural resource“ und „for the benefit of mankind“ sind dem Deklarationstext entnommen, Paragraphen 1 und 3. Siehe auch Nima Nayebi: The Geosynchronous Orbit and the Outer Limits of Westphalian Sovereignty, in: Hastings Science \& Technology Law Journal 3 (2011), 2, 471-498. 
der Polgebiete und des Mondes als Niemandsland die Geschichte der imperialen Aneignung Amerikas, Australiens, Indonesiens und Afrikas fort. Wieder wurde die Abwesenheit von Besitzrechten einseitig durch die Kolonialmacht erklärt. In manchen Fällen, wie etwa in der Arktis, wurden Gebiete als unbewohnt deklariert und auf diese Weise ihrer indigenen Bevölkerungen entledigt; sie wurden buchstäblich entleert und zu Niemandsland gemacht. ${ }^{13}$

Der Nordpol war seit dem 19. Jahrhundert symbolisch umkämpft, ein Ort der Sehnsucht und des Abenteuers in der heroischen europäischen Expeditionskultur. Doch ging es auch um Fisch- und Wildbestände und um Erze, Silber und Gold, als die jungen Nationalstaaten Europas und Nordamerikas die Regionen nördlich des Polarkreises in Beschlag nahmen und systematisch ausbeuteten, oftmals ohne Rücksicht auf die indigenen Bevölkerungen. Das wohl einschlägigste Beispiel für einen Vergleich nationaler Interessen, geopolitischer Zusammenhänge und internationaler Zugeständnisse im Umgang mit arktischem Gebiet ist Spitzbergen. Die karge Inselgruppe im Arktischen Ozean galt als Niemandsland im besten Sinne, unbewohnt, unwirtlich und uninteressant, bis dort Kohlevorkommen entdeckt wurden. Im Zuge der europäischen Hochindustrialisierung an der Wende zum 20. Jahrhundert trieben Einzelunternehmen vor allem aus den Niederlanden, den USA, England, Russland, Schweden und Norwegen die effektive Besetzung Spitzbergens voran, indem sie Gelände annektierten, Kohlebergwerke eröffneten, Werkssiedlungen einrichteten, Infrastrukturen aufbauten und Forschung betrieben. Existierten die Grubenanlagen unterschiedlicher Nationalitäten zunächst nebeneinander, verschärfte der europäische Kohle-Rausch Anfang des 20. Jahrhunderts die Konkurrenzsituation. Die Weltwirtschaftskrise der 1920er Jahre ließ viele Gruben unrentabel werden. Die Gruben wurden geschlossen oder an Norwegen verkauft und das internationale wirtschaftliche Interesse an Spitzbergen erlosch weitgehend. 1920 erzielten die auf Spitzbergen vertretenen Staaten eine Einigung über die Inselgruppe in Form des Spitzbergenvertrags, den sie im Vertragswerk des Völkerbundes registrieren ließen. Die Unterzeichnerstaaten erkannten Spitzbergen als ein prinzipiell allen zugängliches und wirtschaftlich offenes Gebiet an. Die friedliche ökonomische und wissenschaftliche Nutzung sollte garantiert, der Rohstoffabbau jedoch reguliert werden. Dazu wurde Spitzbergen unter norwegische Verwaltung gestellt. Der Spitzbergenvertrag erkannte die Staatshoheit Norwegens über die Inselgruppe an, um „diese Gebiete im Ge-

13 Oran R. Young: Governing the Arctic. From Cold War Theater to Mosaic of Cooperation, in: Global Governance 11 (2005), 1, 9-15. 
nuss einer Verwaltungsform zu wissen, die der Billigkeit entspricht und geeignet ist, ihre friedliche Verwertung und Nutzbarmachung zu verbürgen“".14

Der Kampf um die Kohle auf Spitzbergen war immer auch ein politisches Mittel im Kampf um die Vorherrschaft der arktischen Anrainerstaaten im Norden. Das Nordpolarmeer und der Untergrund des Polareises selbst galten nicht als Kontinent, sondern als zumeist gefrorener Ozean, der mit den Abkommen über die Freiheit der Meere abgedeckt und aufgrund seiner Unwirtlichkeit lange Zeit wirtschaftlich uninteressant schien. Bis in die 1970er Jahre hinein herrschte zudem die Meinung vor, dass der Arktische Ozean kälter werden und das Polareis weiter expandieren würde, was dazu führte, dass das Polareis erst nach der Hochzeit der arktischen Kolonialisierung in das Fadenkreuz der internationalen Debatten um Eigentums- und Nutzungsrechte rückte. Das Ende des Kalten Krieges und die rasante Polschmelze des letzten Jahrzehnts erbrachten diese Wende. Das schmelzende Eis wurde zum Synonym für die Risiken des globalen Klimawandels, aber auch für neue wirtschaftliche Chancen durch die Erschließung neuer Wasserwege, Fischgründe, Erz- und Energievorkommen, Wald- und Wildbestände sowie die Öffnung des Gebiets für den Tourismus. ${ }^{15}$

Symbolisch repräsentiert ist das nationale Rennen um die arktischen Ressourcen in dem Akt Russlands, im Jahre 2007 mittels eines bemannten Tauchboots die russische Flagge unter das Eis auf den Nordpol zu setzen. Demgegenüber stehen gegenwärtige Bestrebungen von Vertretern nicht-arktischer Staaten wie Indien und China, die Arktis als globales Gemeinschaftsgut, als global commons, zu deklarieren, indem sie nachhaltige Entwicklung und Umweltschutz als „,common Arctic issues “ proklamieren. ${ }^{16}$ Die damit verbundenen Ansprüche sind allerdings nicht unbedingt weniger strittig und exklusiv als die nationalen Nutzungsansprüche, denn auch sie versuchen in erster Linie, Teilhabe zu sichern. Sie führen je-

14 Vertrag über Spitzbergen, unterzeichnet zu Paris am 9. Februar 1920, deutsche Übersetzung von 1925 zum „Entwurf eines Gesetzes über den Beitritt Deutschlands zum Spitzbergenvertrage“, in: Verhandlungen des Deutschen Reichstags und seiner Vorläufer. http://www.reichstagsprotokolle.de/index.html (17. März 2014). Siehe auch Dag Avango/Annika E. Nilsson/Peder Roberts: „Assessing Arctic Futures. Voices, Resources and Governance“, in: The Polar Journal (2013), 431-446.

15 Klaus Dodds: Flag Waving and Finger Pointing. The Law of the Sea, the Arctic and the Political Geographies of the Outer Continental Shelf, in: Political Geography 29 (2010), 2, 63-73; Carina Keskitalo: Negotiating the Arctic. The Construction of an International Region. New York u.a. 2004. 16 Zitat aus Punkt 1 der sogenannten Ottawa Deklaration von 1996, mit der sich der Arctic Council, das Forum der acht arktischen Anrainerstaaten, formal etablierte. „Declaration on the Establishment of the Arctic Council Ottawa 1996“, in: Arctic Council. http://www.arcticcouncil.org/index.php/en/document-archive/category/4-founding-documents\# (17. März 2014). 
doch neue Motive wie Umweltsicherheit und Umweltgerechtigkeit ins Feld, die geteilte globale Problem- und Interessenlagen unterstellen. Mit Bezug auf ein verbindendes „wir“, das gemeinsame Weltsichten und Bedürfnisse der Menschheit nahelegt, reihen sich auch solche Personen, Organisationen und Staaten, die geographisch weder als arktisch noch als subarktisch gelten, in den weiteren Kreis der arktischen Stakeholder ein. ${ }^{17}$

Die Arktis bildet nur ein Beispiel für die verschiedenen möglichen diskursiven und performativen Zuschnitte eines Raumes zwischen Niemandsland und Gemeindeland. Die Vergemeinschaftung von terrestrischem und extraterrestrischem Raum als (globales) Gemeinschaftsgut oder (global) commons folgte und folgt dem natur- und völkerrechtlichen Prinzip des res communis (omnium), wie es Grotius für die hohe See ausgearbeitet hatte. Einschlägig ist etwa der Anspruch des kleinen Mittelmeerstaates Malta, der in Person seines UN-Botschafters Arvid Pardo der UN-Vollversammlung im Jahre 1967 vorschlug, den Boden der Weltmeere außerhalb nationalstaatlicher Hoheitsgewässer zum Gemeingut zu deklarieren, das ausschließlich friedlichen Zwecken vorbehalten bleiben und dessen natürliche Rohstoffe ausschließlich dem allgemeinen Interesse der Menschheit dienen sollten. Pardo bezog sich direkt auf das Prinzip des res omnium communis in der Tradition der Freiheit der Meere, knüpfte das Prinzip aber an ein internationales Regime, das nur durch die UN gewährleistet werden könne. ${ }^{18}$ Das Verständnis von gemeinschaftlichem Eigentum und gemeinschaftlicher Nutzung wiederum weitete die Tradition der kollektiven Bewirtschaftung von Gemeindeflächen auf die internationale Gemeinschaft aus. Der englische Begriff common meint sowohl (all)gemein als auch gemeinschaftlich. Die Commons bezeichneten und bezeichnen entsprechend das Gemeingut und das daraus abgeleitete Gemeinwohl.

Als älteste Beispiele für die Praxis der Commons-Bewirtschaftung werden zumeist die Gemeindeflächen des mittelalterlichen englischen Dorfes angeführt der Dorfbrunnen, der Dorfteich, die Dorfweide oder der umliegende Forst. Die Allmende wurde traditionell kollektiv genutzt, um alltägliche Grundbedürfnisse zu stillen: Wasser zu holen, zu fischen, Feuerholz zu sammeln oder das Vieh zu weiden. In einem ähnlichen Sinne betonten die Vertreter eines Prinzips der global

17 Paul Arthur Berkman: Our Common Future in the Arctic Ocean, in: The Round Table. The Commonwealth Journal of International Affairs 101 (2012), 2, 123-135; Peter J. Taylor: How Do We Know We Have Global Environmental Problems? Undifferentiated Science-Politics and Its Potential Reconstruction, in: Peter J. Taylor/Saul E. Halfon/Paul N. Edwards (Hg.): Changing Life. Genomes, Ecologies, Bodies, Commodities. Minneapolis 1997, 149-174.

18 Arvid Pardo: „Rede vor der United Nations General Assembly, 1. November 1967“, in: Oceans and Law of the Sea United Nations. http://www.un.org/depts/los/convention_agreements/ texts/pardo_ga1967.pdf (17. März 2014). 
commons in den 1970er Jahren, wie Pardo, die Auskömmlichkeit von und den gerechten Zugang zu lebenswichtigen irdischen Ressourcen wie Luft und Wasser. Räume, die natürliche Prozesse in der Biosphäre aufrechterhielten wie die Meere oder die Atmosphäre, aber auch solche Räume, die infrastrukturell erschlossen waren, so wie der Luftraum und der Weltraum außerhalb der Grenzen nationaler Souveränität, sollten dem Diktum Grotius' folgend „common to all and proper to none" bleiben. ${ }^{19}$

\section{Schutz- und Nutzprinzip}

Wäre 1970 demnach ein Commons-Moment gewesen? Gleich welche Begrifflichkeit verwendet wurde, ob die Allmendefläche der commons, die juristische Kategorie des res communis oder schließlich das Prinzip des Common Heritage of Mankind, des gemeinsamen Menschheitserbes: Das Commoning verteilte Verantwortlichkeiten und Verfügungsrechte nicht zuerst gemäß physischer Präsenz, geographischer Nähe oder technischem, militärischem und ökonomischem Vermögen, sondern entlang neuer partizipatorischer Grundsätze, die auch die Bedürfnisse der Länder der sogenannten sich entwickelnden Welt des globalen Südens gegenüber dem privilegierten Norden anerkennen wollten. Pardo gilt mit seiner Rede als der Begründer des CHM-Prinzips als rechtlicher Figur, obwohl er selbst die World Peace through Law Konferenz in Neu Delhi vom Juli 1967 als Urheber des Konzepts zitierte, um zu erklären:

The sea-bed and the ocean floor are a common heritage of mankind and should be used and exploited for peaceful purposes and for the exclusive benefit of mankind as a whole. The needs of poor countries, representing that part of mankind which is most in need of assistance, should receive preferential consideration in the event of financial benefits being derived from the exploitation of the sea-bed and ocean floor for commercial purposes. ${ }^{20}$

Der Diskurs über die global commons eröffnete die Möglichkeit, Gebiete auf der Basis und in Hinsicht auf neue transnationale und globale Anliegen wie der Ressourcen- und Umweltproblematik zu vergemeinschaften. Zugleich lenkten die modernen Technowissenschaften der Ozeanographie und Tiefseeforschung, der Weltraumforschung und Polarforschung erst die Aufmerksamkeit auf die Erde und das Weltall als erschließbare und ertragreiche natürliche Ressourcen. ${ }^{21}$ Räu-

19 Nil Disco/Eda Kranakis (Hg.): Cosmopolitan Commons. Sharing Resources and Risks across Borders. Cambridge, Mass. 2013.

20 Pardo, Rede vor der United Nations General Assembly, 1. November 1967, Paragraph 13. 
me wie der Meeresboden, die Polargebiete und das Weltall sollten nicht primär geschützt, sondern genutzt werden. Die Idee eines Common Heritage of Mankind warb nicht nur für ein neues Schutzprinzip, sondern auch und vor allem für ein neues Nutzprinzip.

Geschützt werden sollten die infrage stehenden Gebiete zunächst vor allem vor dem militärischen und dem ökonomischen Zugriff einzelner mächtiger Staaten und Unternehmen. Wie im Falle Maltas waren es oft die Staaten des Westens selbst, die in Reaktion auf neue technologische Zugriffsmöglichkeiten versuchten, das politische und ökonomische Weltgefüge und ihren Platz darin stabil zu halten. Die ersten Vertragswerke über gemeinschaftlichen Besitz waren sämtlich sicherheitspolitisch motiviert. Es waren Abkommen zur Rüstungskontrolle wie das Antarktische Vertragswerk (Antarctic Treaty System 1959), der UN-Vertrag über das Verbot einer Stationierung von Waffen auf dem Meeresboden (1970) oder das frühe internationale Gesetzeswerk zum Weltraum, das durch das UN Committee on the Peaceful Use of Outer Space ausgearbeitet wurde. ${ }^{22}$ Bereits kurz nach dem erfolgreichen Start des sowjetischen Satelliten Sputnik im Jahre 1957 wurde das Weltraumvertragswerk zuerst diskutiert. Die erste Einigung über ein Verbot von Nukleartests im Weltraum wurde 1963 zwischen den USA, der Sowjetunion und Großbritannien abgeschlossen. Der UN-Weltraumvertrag von 1967 (UN Outer Space Treaty 1967) verbot jegliche militärische Aktivität im All und beschränkte dessen Erforschung und Nutzung auf wissenschaftliche Zwecke. Er untersagte Regierungen (insbesondere den USA und der UdSSR, später auch Unternehmen und Privatpersonen), einen Himmelskörper zu besetzen, auszubeuten oder als Deponie für radioaktive Abfälle zu gebrauchen. Die Nutzung des Weltraums, so hieß es, sei „im gemeinsamen Interesse“ und „zum Vorteil aller Länder“ und daher der Verfügungsgewalt aller zu unterstellen. ${ }^{23}$ Doch noch wäh-

21 Alexandre Kiss: The Common Heritage of Mankind. Utopia or Reality?, in: International Journal 40 (1985), 3, 423-441.

22 „Antarctic Treaty (1. Dezember 1959, Washington, D.C.; trat 1961 in Kraft)“, in: National Science Foundation: Directorate for Geoscience (GEO). http://www.nsf.gov/od/opp/antarct/ anttrty.jsp (17. März 2014). Der Antarktisvertrag kam als Teil des IGY 1957/1958 zustande. Er wurde nicht unter der Ägide der Vereinten Nationen geschlossen, sondern im Rahmen einer Vereinbarung von zwölf Unterzeichnerstaaten der Antarktiskonferenz 1959. Der Vertrag wurde wissenschaftlich koordiniert durch das Komitee SCAR - Scientific Committee on Antarctic Research: UN General Assembly Resolutions: Treaty on the Prohibition of the Emplacement of Nuclear Weapons and Other Weapons of Mass Destruction on the Seabed and the Ocean Floor and in the Subsoil Thereof (7. Dezember 1970); Treaty on Principles Governing the Activities of States in the Exploration and Use of Outer Space, including the Moon and Other Celestial Bodies (19. Dezember 1966).

23 Die Zitate „common interest of all mankind“ und „for the benefit and in the interests of all countries“ sind der Präambel und dem Artikel 1 des Weltraumvertrages entnommen. Siehe Uni- 
rend die Vertragsverhandlungen bei den Vereinten Nationen geführt wurden, lieferten sich die USA und die UdSSR ein heißes Rennen um den ersten Platz auf dem Mond.

In keinem der beschriebenen Fälle standen die Umwelt, Sicherheit oder Zukunft des betreffenden Gebiets im Zentrum des Interesses. Es ging um die Umwelt, die Sicherheit und die Zukunft derjenigen Orte, an denen die Akteure positioniert waren. Gleichwohl wurden die dominanten Interessen einzelner Akteure einem proklamierten gemeinsamen Interesse aller untergeordnet, das ressourcen-, sicherheits- und umweltpolitisch begründet wurde. In der internationalen Debatte um die Weltmeere galten die Ozeane als eine scheinbar unermessliche Quelle tierischer und mineralischer Rohstoffe und als Motor für die klimatischen Prozesse der Erde, zunehmend aber auch als Senke, als marine Endlagerstätte für Abfälle. Neben der Überfischungsproblematik geriet die zunehmende Verschmutzung durch Verklappung auf die internationale Agenda. Die Convention on the Prevention of Marine Pollution by Dumping Wastes and Other Matter (die sogenannte London Dumping Convention) wurde durch eine interstaatliche Konferenz in London ausgearbeitet und fand weltweit Anerkennung. Sie wurde im November 1972 verabschiedet und trat 1975 in Kraft. ${ }^{24}$

Während im Weltraumvertrag noch keine Rede vom Weltraum und von fernen Planeten als Erbe der Menschheit war, formulierte die Erklärung über die Nutzung des Meeresbodens drei Jahre später, 1970, explizit:

The seabed and ocean floor, and the subsoil thereof, beyond the limits of national jurisdiction [...], as well as the resources of the area, are the common heritage of mankind. ${ }^{25}$

Im Seerecht wurde der Begriff des Gemeinsamen Erbes der Menschheit zuerst artikuliert und er war geschickt gewählt. Das Menschheitserbe verknüpfte das Gemeingut und das Gemeinwohl der Commons mit dem neuen Bild der ganzen Erde und des sie umgebenden Weltraums als eines zu wahrenden Vermögens.

ted Nations Treaties and Principles on Outer Space, Text of Treaties and Principles Governing the Activities of States in the Exploration and Use of Outer Space, Adopted by the United Nations General Assembly. New York 2002, 3-4; siehe auch J.E. S. Fawcett: Outer Space. New Challenges to Law and Policy. Oxford 1984.

24 „Convention on the Prevention of Marine Pollution by Dumping of Wastes and Other Matter, 1972 (in Kraft getreten 1975)“, in: IMO International Martime Organization. http://www.imo.org/ about/conventions/listofconventions/Pages/Default.aspx (17. März 2014).

25 „UN General Assembly Resolution: Declaration of Principles Governing the Seabed and the Ocean Floor, and the Subsoil Thereof, beyond the Limits of National Jurisdiction (7. Dezember 1970), Art. 1“, in: United Nations: Dag Hammarskjöld Library. http://www.un.org/depts/dhl/ resguide/r25_en.shtml (17. März 2014). 
Das Konzept war anthropozentrisch, denn es betrachtete diese Räume, gleich ob als zu schützende oder auszubeutende Gebiete, in ihrem instrumentellen Wert für den Menschen. ${ }^{26}$ Das Erbe übertrug die Verantwortung (stewardship) des Schutzes und der Nutzung den gegenwärtigen menschlichen Generationen. Es setzte sie in eine Beziehung zu ihren Vorfahren, als in deren Schuld stehend, und zu ihren Nachkommen, den zukünftigen Generationen, als für sie in die Pflicht tretend. Lange bevor Nachhaltige Entwicklung 1987 zum globalen Programm erhoben wurde, vermittelte das Menschheitserbe eine Vorstellung sozialer Nachhaltigkeit im Umgang mit irdischen Ressourcen. Es begegnete der vorherrschenden ökonomischen Logik der Verzinsung, die in der Zukunft liegende Ereignisse und Vermögen systematisch diskontierte. Der westlichen Kapitalund Ressourcenpolitik der Abzinsung, der zufolge ein gegenwärtiger Kapitalbestand als ökonomisch wertvoller galt als derselbe Bestand in der Zukunft, trat der Erbebegriff entgegen, indem er die Zukunft aufwertete. Den zukünftigen Generationen maß er einen Eigenwert in der Gegenwart bei, denn nicht das einzelne wohlhabende Geschlecht, sondern die ganze Menschheit sollte in ihrem Fortbestand gesichert werden. ${ }^{27}$

Das Schutzprinzip des Common Heritage of all Mankind tauchte bereits 1954 in der Haager Konvention auf, doch diese Konvention schützte Kulturgut. Die UNESCO-Welterbekonvention von 1972 schützte sowohl Kultur- als auch Naturerbestätten, jedoch gemäß Artikel 2 nur „genau abgegrenzte Gebiete“: „Naturgebilde“, „geologische und physiographische Erscheinungsformen“ und „Naturstätten“. ${ }^{28}$ Die Programmatik, große Teile der Erde als gemeinsames Er-

26 Kemal Baslar: The Concept of the Common Heritage of Mankind in International Law. The Hague 1998.

27 Stefan Willer: Nachhaltige Zukunft: Kommende Generation und ihr kulturelles Erbe, in: Heinrich Hartmann/Jakob Vogel (Hg.): Zukunftswissen. Prognosen in Wirtschaft, Politik und Gesellschaft seit 1900. Frankfurt a.M. u.a. 2010, 267-283; Edith Brown Weiss: In Fairness to Future Generations. International Law, Common Patrimony, and Intergenerational Equity. New York 1989; Volker Hauff (Hg.): Unsere Gemeinsame Zukunft. Der Brundtland-Bericht der Weltkommission für Umwelt und Entwicklung. Greven 1987.

28 "Convention for the Protection of Cultural Property in the Event of Armed UNESCO Legal Instruments“. http://portal.unesco.org/en/ev.php-URL_ID=13637\&URL_DO=DO_TOPIC\&URL_ SECTION=201.html (17. März 2014); „UNESCO-Welterbekonvention 1972“, in: UNESCO: Deutsche UNESCO-Kommission e.V. http://www.unesco.de/welterbe-konvention.html (17. März 2014); Andrea Rehling: Universalismen und Partikularismen im Widerstreit. Zur Genese des UNESCOWelterbes, in: Zeithistorische Forschungen/Studies in Contemporary History, Online-Ausgabe, 8 (2011), 3. http://www.zeithistorische-forschungen.de/site/40209179/default.aspx (17. März 2014); Mechtild Rössler: Weltkulturerbe und Globalisierung. Vom Weltwunder zum Erbe der Menschheit, in: Iris Schröder/Sabine Höhler (Hg.): Welt-Räume. Geschichte, Geographie und 
be verbindlich zu regeln und vor einer expansiven imperialen Besetzung und Ausbeutung zu schützen, demonstrierte, wie gewaltig die Befürchtungen und Hoffnungen waren, die während des Kalten Krieges mit diesen Gebieten verknüpft wurden. In den westlichen Gesellschaften der 1960er Jahre standen dem boomenden Fortschrittsoptimismus des Raumfahrtzeitalters die Wahrnehmung wachsender globaler Interdependenzen sowie die Sorge um exponentiell verlaufende Wachstums- und Schrumpfungsprozesse von Weltbevölkerung, Ressourcen und Umweltqualität gegenüber. Der UN-Botschafter der USA, Richard Newton Gardner, fasste die Lage zur Begrenztheit und fragwürdigen Zukunft des Planeten 1970 so zusammen:

All mankind depends on the same scarce and relatively shrinking resource pool.29

Befürchtungen hinsichtlich der Endlichkeit irdischer Ressourcen und die Notwendigkeit internationaler Abstimmungen waren seit der imperialen Aufteilung der Erde geäußert worden - insbesondere von solchen Staaten, die sich irdischen Raum in der Form nationalen Territoriums angeeignet und demzufolge viel zu verlieren hatten. Während die nationale Besetzung und Zuordnung von irdischem Festland im Vordergrund ihrer Sorge standen, wurden mit den Globalisierungsprozessen des 19. Jahrhunderts auch immaterielle Ressourcen mittels wissenschaftlich-technischer Konventionen zurechtgeschnitten, um sie für die komplizierte internationale Mechanik der europäischen Mächte in kompatible Formen zu bringen. Beispiele hierfür sind die Einigung auf den Goldstandard als internationale Währungsbasis, auf das metrische System 1875 sowie auf die Weltstandardzeit, mit der im Jahre 1884 der Globus in einheitliche Zeitzonen eingeteilt wurde. ${ }^{30}$ Die Anstrengungen, anschlussfähige Informations-, Verkehrsund Handelssysteme zu schaffen, gingen einher mit der Aufteilung der Welt unter den Mächtigen und weniger Mächtigen. Seit der Jahrhundertwende war beispielsweise die Luft nicht mehr für jeden in jeder Hinsicht frei verfügbar. Mit den Erfolgen in der Luftfahrt setzte sich das Prinzip der nationalen Lufthoheit

\footnotetext{
Globalisierung seit 1900. Frankfurt a.M. 2005, 235-257; David Lowenthal: Natural and Cultural Heritage, in: International Journal of Heritage Studies 11 (2005), 1, 81-92.

29 Richard N. Gardner: New Tasks for the United Nations. The Environment, Population, Space, and Seabeds, in: Annals of the New York Academy of Sciences 184 (1971), 352-365.

30 Martin H. Geyer: One Language for the World. The Metric System, International Coinage, Gold Standard, and the Rise of Internationalism, 1850-1900, in: Martin H. Geyer/Johannes Paulmann (Hg.): The Mechanics of Internationalism. Culture, Society and Politics from the 1840s to the First World War. Oxford 2001, 55-92; Peter Galison: Einstein's Clocks, Poincaré's Maps. Empires of Time. London 2004.
} 
gegen das Prinzip der Luftfreiheit durch und die dritte Dimension wurde zum internationalen Rechtsobjekt. In dieses Feld des Äthers gehört auch die Verteilung des Radiofrequenzspektrums unter den europäischen Nationen und ihren Sendern im frühen 20. Jahrhundert. ${ }^{31}$

Die Protagonisten der Gliederung von Raum und Zeit des späten 19. Jahrhunderts waren als Vertreter der Nationalstaaten, ob aus Wissenschaft, Wirtschaft oder Politik, zuvorderst an einer Vereinheitlichung im Sinne funktionaler und nützlicher globaler Infrastruktursysteme interessiert. Sie zogen die geteilte der gemeinschaftlichen Nutzung der Erde vor. Ihre Kompromisse in der Verteilungsfrage suchten die nationale Vorrangstellung in einer immer stärker verflochtenen Welt zu sichern. ${ }^{32}$ Obwohl die Vorstellung, irdische und außerirdische Räume unter dem CHM-Prinzip schützen und nutzen zu können, in vielerlei Hinsicht Parallelen zu den Praktiken des späten 19. Jahrhunderts aufweist, kann sie doch in einer Hinsicht als beispiellos in ihrem Anspruch angesehen werden: Die Unternehmung versprach nichts weniger als die ,geerbte“ Erde und eine globale Umwelt für zukünftige Generationen treuhänderisch zu verwalten, indem sie die Menschheit selbst als Souverän einsetzte. Es war aber, wie im folgenden Abschnitt weiter auszuführen sein wird, gerade diese Berufung auf höhere Prinzipien - auf die ganze Erde und auf die Menschheit als Betroffene, als Nutznießerin und als Verwalterin gleichermaßen -, die das CHM-Prinzip auf produktive Weise uneindeutig werden ließ, so dass es teilweise gegenläufige Motive und Interessen unter sich vereinbaren konnte.

\section{Individualismus und Kollektivismus}

Zweifellos lag eine enorme performative Kraft in den Begriffen common, heritage und mankind. In Zeiten der weltpolitischen Erstarrung schienen sie der menschlichen Existenz einen neuen Sinn in der Übernahme der Verantwortung für den Planeten zu verleihen. Doch sagen diese Termini weniger über den geeinigten oder geteilten Zustand der Welt um 1970 als über die Anlässe und Möglichkeiten verschiedener nationaler und internationaler Akteure, Organisationen und Netzwerke der Zeit, ,gemeinsame‘ Werte, Interessen, Ansprüche und Verantwortlich-

31 Natalie Lübben: Das Recht auf freie Benutzung des Luftraums. Berlin 1993; Nina Wormbs: Technology-dependent Commons. The Example of Frequency Spectrum for Broadcasting in Europe in the 1920s, in: International Journal of the Commons 5 (2011), 1, 92-109.

32 Charles S. Maier: Leviathan 2.0. Inventing Modern Statehood, in: Emily S. Rosenberg (Hg.): A World Connecting, 1870-1945. Cambridge, Mass. 2012, 27-282. 
keiten überhaupt erst zu definieren. ${ }^{33}$ Der Fall des Arktischen Ozeans, der erst seit wenigen Jahren als ein potenzielles CHM-Gebiet international verhandelt wird, demonstriert, dass Räume immer dann als Gemeinschaftsgut interessant wurden, wenn ihre Nutzung technisch projektierbar und profitabel schien. Wie die Territorialisierung wurde auch die Vergemeinschaftung von terrestrischen und extraterrestrischen Räumen als ein aktiver Prozess betrieben, um Ressourcen im eigenen Interesse und zum eigenen Vorteil als globale Gemeinschaftsgüter zu deklarieren. Es waren nicht nur, aber auch die sich entwickelnden Staaten, die sich für diese neue Form der Teilhabesicherung auf dem internationalen Parkett stark machten. Sie sollten jedoch auf die enorme Skepsis des Westens gegenüber kollektiven Formen des Wirtschaftens treffen.

Die Kontroversen um Vergemeinschaftung und Nationalisierung um 1970 spiegeln sich etwa im langwierigen Streit um die Nutzung des Meeresbodens, von den Genfer Seerechtskonventionen 1958 über die erste UN-Deklaration über die Nutzung des Meeresbodens 1970 bis zur Unterzeichnung des UN-Seerechtsabkommens 1982. Um die Ausdehnung der Küstengewässer und um die Festlandsockelregelung wurde heftig gerungen. Erst 1994 waren die Haupthindernisse für die Ratifizierung und das Inkrafttreten des Abkommens ausgeräumt. Die USA jedoch traten dem Abkommen nie bei, sondern behielten sich vor, den Genfer Konventionen $\mathrm{zu}$ folgen, die das Gewohnheitsrecht kodifizierten. ${ }^{34}$ In dem Für und Wider über hoheitlich und gemeinschaftlich genutzte Gewässer verwischten sich die jeweils zugrunde gelegten Eigentums- und Nutzungsprinzipien auf eine Weise, die zu verstehen gibt, warum bei aller Gemeinschaftsrhetorik die CommonsPrinzipien weniger unter den Grundsätzen des terra communis, sondern unter territorialen Prinzipien diskutiert und in die internationale Rechtsprechung eingelagert wurden. Der amerikanische Humanökologe Garrett Hardin, der vor allem durch seine provozierenden Thesen im zeitgenössischen Diskurs der Überbevölkerung bekannt wurde, fachte 1968 die Commons-Debatte weiter an, als er in einem provokanten Artikel The Tragedy of the Commons (Die Tragödie der Allmende) in der Zeitschrift Science die Commons öffentlich als ein irrationales und rückwärts gewandtes Konzept aburteilte. Aus der Sicht der Naturwissenschaften sei die herrschende Politik des laissez-faire in Bezug auf das Bevölkerungswachstum eine gedankenlose Politik der Commons, die zur ungezügelten Nutzung und Übernutzung irdischer Ressourcen führe und daher zum Scheitern verurteilt sei. Wie so viele seiner Zeitgenossen missverstand Hardin die Commons beziehungsweise die traditionelle Dorfweide als terra nullius, als ein herrenloses Gebiet, das

33 Paul B. Hartzog: Global Commons. Is Definition Possible? Salt Lake City 2003.

34 Martin Ira Glassner (Hg.): The United Nations at Work. Westport 1998. 
durch rationale Individuen systematisch und zwangsläufig bis zur Überweidung ausgenutzt werden würde. ${ }^{35}$

Kapitalismus, Sozialismus, Commonismus und Philanthropie - in seinem schematischen Vergleich von vier Wirtschaftssystemen und ihrem Umgang mit natürlichen Ressourcen meinte Harding nachweisen zu können, dass hinsichtlich der Verantwortlichkeit und Haftbarkeit der Nutzer die gruppenzentrierten Ökonomien deutlich schlechter abschnitten als die egozentrierten Ökonomien. An jedes der Systeme richtete er die Fragen, erstens, wessen Vieh dort weide, und zweitens, wer die Erträge aus der Viehwirtschaft ernte. In einem privatwirtschaftlich organisierten System sei dies in beiden Fällen das investierende Individuum, im Sozialismus in beiden Fällen das Kollektiv. Diese Ökonomien seien insofern fair, als die Chancen und Risiken des Wirtschaftens jeweils auf dieselben Akteure verteilt seien. Die philanthropische oder altruistische Ökonomie kranke daran, dass das Individuum investiere, während die Gemeinschaft profitiere. Als besonders problematisch aber zeigte sich Hardin zufolge das Commons-System, denn hier bringe die Gemeinschaft die Ressource ein, während allein das Individuum profitiere. ${ }^{36}$

Dieses Missverhältnis offenbare sich, sobald die Gemeindeflächen übernutzt würden. Die Dorfweide bildete Hardins Metapher der gemeinschaftlich genutzten irdischen Ressourcen wie Wälder und Meere, deren ökologische Tragfähigkeit so überstrapaziert werde, dass die globalen Holz- oder Fischbestände langfristig ausgeplündert würden. Wieder stellte Hardin die Frage, wer in diesem Falle gewinne und verliere beziehungsweise welches System die höchste intrinsische Verantwortung aufweise. Und erneut ging das privat- oder marktwirtschaftliche System als eindeutiger Sieger aus dem Wettstreit hervor. Dessen Verantwortung drücke sich in einem Verhältnis von Sorge und Vorsorge aus und ähnlich ließe sich für die auf das Kollektiv ausgerichtete Wirtschaftsform des Sozialismus argumentieren. Ein Commons-System hingegen bevorteile den gewissenlosen Nutzer, der seinen eigenen Gewinn mehre, während sich die Verluste auf die Gruppe verteilten. Es sei gleichsam im Interesse des Einzelnen, so Hardins Schlussfolgerung, egoistische Entscheidungen auf Kosten der Gemeinschaft zu treffen. Die Commons begüns-

35 Garrett Hardin: The Tragedy of the Commons, in: Science 162 (1968), 13, 1243-1248; Sabine Höhler: The Law of Growth. How Ecology Accounted for World Population in the 20th Century, in: Distinktion. Scandinavian Journal of Social Theory 14 (2007), Themenheft „Bioeconomy“, 4564.

36 Garrett Hardin: Exploring New Ethics for Survival. The Voyage of the Spaceship Beagle. New York 1972, 109ff. 
tigten ein rücksichtsloses, ressourcenintensives und ausbeutendes Wirtschaften auf Kosten anderer bis zum Ruin aller. So lautete sein bekanntes Fazit:

Freedom in a commons brings ruin to all. Inevitably. Remorselessly.“37

Hardins Systemvergleich war in vielerlei Hinsicht problematisch. Die von ihm geprüften Wirtschaftsformen bezogen sich zwar alle auf das Gemeinschaftsgebiet der Allmende, doch konstruierten sie diese Allmende in ganz unterschiedlicher Weise. In der privatwirtschaftlichen Nutzung ging Hardin von der Möglichkeit einer individuell aufgeteilten und eingezäunten Dorfweide aus, doch waren große Teile der globalen Umwelt, wie Hardin selbst an anderer Stelle über die Weltmeere bemerkte, keineswegs einfach aufteilbar: „It is doubtful if we can create territories in the ocean by fencing. “" ${ }^{38}$ Die nicht eingezäunte Natur war zu Hardins Zeit mitnichten in eine umweltverantwortliche Marktwirtschaft integriert. Allmenden wie Luft und Gewässer wurden privatwirtschaftlich genutzt, ohne dass die Kosten ihres Verbrauchs und ihrer Verschmutzung in den Markt und in die Preisgestaltung integriert worden wären. ${ }^{39}$ Auf diesen Umstand und seine Konsequenzen in der Form vergifteter Bäche und Böden bezog sich die in den 1960er Jahren aufkommende amerikanische Umweltbewegung, ausgelöst durch Rachel Carsons Buch Silent Spring von 1962 über die fatalen Umweltfolgen des Einsatzes von DDT als Insektizid in der Landwirtschaft. ${ }^{40} \mathrm{Als}$ eine explizit politische Kategorie wurde Umwelt in staatlichen Umweltschutzeinrichtungen und in Maßnahmen wie der 1970 gegründeten US-amerikanischen Environmental Protection Agency (EPA) repräsentiert. ${ }^{41}$ Der Umweltbegriff trug dem anthropogenen Charakter der modernen Naturgestaltung Rechnung. Der Umweltschutzlöste den konservativ ausgelegten Naturschutz in den USA und Europa ab, der seit dem späten 19. Jahrhundert Natur als das Andere der modernen Zivilisation verstand und ihren vermeintlich ursprünglichen Charakter der Wildnis oder pastoralen Landschaft vor Zerstörung bewahren wollte.

Bis 1970 war es das politisch geförderte privatwirtschaftliche System, das die Ausplünderung kollektiver Ressourcen erlaubte und begünstigte. Es umschrieb

37 Hardin, Exploring New Ethics (1972), 118.

38 Ebd., 121.

39 In den 1960er Jahren entwickelte und propagierte die entstehende ökologische Ökonomik neue Instrumente zur Internalisierung von Umweltkosten in die volkswirtschaftliche Gesamtrechnung. Siehe Inge Røpke: The Early History of Modern Ecological Economics, in: Ecological Economics 50 (2004), 293-314.

40 Rachel Carson: Silent Spring. Boston 1962.

41 Jens Ivo Engels zufolge tauchte der Begriff „Umweltschutz“ in der BRD als Quellenbegriff der politischen Debatte erst 1969 auf. Ders.: Naturpolitik in der Bundesrepublik. Ideenwelt und politische Verhaltensstile in Naturschutz und Umweltbewegung 1950-1980. Paderborn 2006, 21. 
eben das Commons-System, das Hardin der systematischen Misswirtschaft und des Raubbaus bezichtigte. Hardins Commons-System wiederum beschrieb zwar die kollektive Ressource, es vergemeinschaftete aber deren Nutzung nicht: Das Vieh, das Hardin auf der Gemeindewiese weiden ließ, verblieb im Privatbesitz. Damit machte er eine spezifische Commons-Vorstellung stark: Die Vorstellung einer Ressource in kollektivem Besitz, die individuell genutzt und schließlich vernutzt wird. Hardin sprach damit mitnichten von den traditionellen Commons, sondern von Niemandsland als demjenigen Verfügungsprinzip, das Ressourcen auf einer first-come-first-serve-Basis verteilte. Es war das Prinzip, mit dem die hochindustrialisierten Staaten des Westens historisch bestens vertraut waren. Dieses Prinzip beruhte auf einer Ökonomie der Ungleichheit, die wirtschaftlich, technisch und militärisch einflussreiche Staaten bevorzugte. Der UN-Mondvertrag von 1979 stellt ein Beispiel für die zeitgenössische Macht des terra-nullius-Prinzips über Pläne kollektiver Bewirtschaftung dar. Mit der ersten Mondlandung im Jahre 1969 durch die Amerikaner und das Aufpflanzen der US-Flagge war der Mond als US-Territorium gleichsam annektiert worden und beschäftigte seit 1972 eine UN-Kommission mit der Besitzfrage. Der Mondvertrag erklärte schließlich den Mond zur entmilitarisierten Zone und seine Bodenschätze zum gemeinsamen Menschheitserbe:

The Moon and its natural resources are the common heritage of mankind.

Artikel 11 des Vertrags schützte den Mond vor der nationalen Besetzung, der privaten Aneignung und der kommerziellen Nutzung. Die UN hatten über alle Aktivität auf dem Mond informiert zu werden und über eine mögliche Ressourcennutzung sollte eine internationale Regierung befinden. In diesen Debatten kam den sogenannten sich entwickelnden Ländern ein deutliches Gewicht zu. Ihr Interesse bestand aber vor allem darin, mögliche mineralische Mondressourcen mit dem CHM-Prinzip gegen das private Unternehmertum des Westens abzusichern, um auf diese Weise das Weltwirtschaftsgefüge nicht zu gefährden, das auf westlichen Rohstoffimporten aus dem globalen Süden und Osten beruhte. Praktisch scheiterte der Vertrag an mangelnder Legitimität. Mit Chile, den Philippinen, Uruguay, den Niederlanden und Österreich fanden sich gerade einmal fünf Staaten bereit, den Vertrag zu ratifizieren und ihn 1984 zumindest formal in Kraft zu setzen. Jedoch erklärte sich keiner der Staaten, die zu dieser Zeit ein bemanntes Weltraumprogramm verfolgten, zur Unterzeichnung bereit. ${ }^{42}$

42 „United Nations, Agreement Governing the Activities of States on the Moon and other Celestial Bodies (1979), Art. 11“, in: United Nations: Office for Outer Space Affairs. http://www.unoosa.org/oosa/SpaceLaw/moon.html (17. März 2014); Michael Listner: The Moon 
Hardins strikte Ablehnung des Commons-Systems entsprach der vorherrschenden Sichtweise vieler seiner Zeitgenossen, die eine biologistische These des Kampfes ums Dasein zugrunde legten, nach der Menschen grundsätzlich danach strebten, ihren eigenen Vorteil zu sichern und zu maximieren. Diese Sichtweise schloss solidarisches oder altruistisches Verhalten prinzipiell aus und vor allem ignorierte sie die historischen Wurzeln weltweiter sozialer Disparitäten durch koloniale und postkoloniale Machtbeziehungen. Vielmehr legte sie die Frage der Verteilung von Ressourcen in die Hände derjenigen Menschen und Länder, die durch ihr rationales Handeln zu Reichtum gelangt und offensichtlich besser als andere in der Lage waren, vernünftige Entscheidungen $\mathrm{zu}$ treffen und klug zu wirtschaften. ${ }^{43}$ Hardins Perspektive brachte das tiefe Misstrauen des Westens und insbesondere der USA gegenüber jeglicher Form der Gütergemeinschaft oder kollektiven Güterbewirtschaftung zum Ausdruck. Wie der Rechtswissenschaftler Scott Shackelford bemerkte, galt in der westlichen Welt die Etablierung von Eigentumsrechten nicht als Problem, sondern als die rationale, da eindeutige Lösung zur Verwaltung einer Ressource, die sich außerhalb nationaler Jurisdiktion befand. Sobald deren Vereinnahmung in Aussicht stand, schienen Verfügungsrechte nötig zu werden, um ihre ,Entwicklung' zu katalysieren. ${ }^{44}$

Traditionell standen die Commons jedoch nie außerhalb eines definierten Eigentumsgefüges. Schon vor der britischen Landreform im 18. und 19. Jahrhundert, mit der die dörflichen Commons in einer Serie von Enclosure Acts neu strukturiert und in Privateigentum überführt wurden, war das Commons-System keineswegs ungesetzlich. Es bezeichnete vielmehr ein komplexes soziales System aus Eigentums- und Nutzungsrechten, sei es in der Form des Besitzes der Herde, die auf der Allmende weidete, sei es in der Person des Lehnsherren und Grundeigners, der traditionell Zugangsrechte an die commoners vergab, oder sei es in Bezug auf das System privater Eigentumsverhältnisse allgemein. Historisch zeichnete sich das Commons-System dadurch aus, dass der Einzelne gerade nicht nach Belieben über die Güter der Allgemeinheit verfügen konnte. In fast paradoxer Weise bezeichneten die Commons einen rechtlichen Status, der immer schon in Bezug

Treaty. Failed International Law or Waiting in the Shadows?, in: The Space Review (2011). http://www.thespacereview.com/article/1954/1 (17. März 2014); Baslar, The Concept of the Common Heritage of Mankind in International Law (1998), 159ff.

43 Diese Ansichten finden sich ausbuchstabiert und operationalisiert in Garrett Hardin: Lifeboat Ethics. The Case Against Helping the Poor, in: Psychology Today 8 (1974), 4, 38-43 und 123-126, sowie in William und Paul Paddock: Famine - 1975! London 1968 [orig. Famine - 1975! America's Decision. Who will Survive? Boston 1967].

44 Scott J. Shackelford: The Tragedy of the Common Heritage of Mankind, in: Stanford Environmental Law Journal 27 (2008), 101-157, hier 120. 
auf bestehende Eigentumsverhältnisse bestimmt war, die Souveränität und Territorialität verknüpften.

Der Antarktisvertrag von 1959 verdeutlicht den Zusammenhang der Verwaltung exterritorialen Gebiets mit souveränen Besitzansprüchen, wenngleich er nicht in der Begrifflichkeit der Commons gefasst war. Das Vertragswerk schränkte die Aktivitäten auf dem südlichen Kontinent ein, vor allem aber war er Möglichkeitsbedingung eines reflektierten Patriotismus: Der Konsens der Vertragsstaaten erlaubte es, die konkurrierenden Gebietsansprüche aufrechtzuerhalten, die in den rund fünfzig Jahren zuvor unter dem terra-nullius-Prinzip erhoben worden waren. Diese Ansprüche wurden mit dem Vertrag nicht geklärt, sondern buchstäblich eingefroren. Die Grundlage der Mitbestimmung war und blieb ein Prinzip der Präsenz: Seit den 1960er Jahren identifizierte das Vertragswerk die Konsultativstaaten nach ihren effektiven permanenten Besetzungsaktivitäten in Form von Forschungsexpeditionen und Forschungsstationen vor Ort. Diese Praxis bevorteilte diejenigen Staaten, die sich die teure Ausrüstung geowissenschaftlicher Forschung in Form von Forschungsschiffen, Basisstationen und Forscherteams leisten konnten. Die Erdwissenschaften der Ozeanographie, Meteorologie und Klimaforschung bildeten so in mehrerlei Hinsicht die Basis für eine neue akzeptierte Territorialpolitik - als nationale Organe und Instrumente der geologischen Kartierung des Terrains wie des schieren Zugegenseins. ${ }^{45}$

\section{„Retrenchment“: Neue Grenzziehungen}

Die diskursive Figur des Gemeinsamen Erbes der Menschheit schaffte in seiner Konzeption und Praxis die traditionellen basierten Prinzipien der Landnahme und der Verfügung über irdischen und außerirdischen Raum nicht ab, sondern sie internationalisierte diese Prinzipien. Die Commons-Prinzipien, die im Antarktiswerk, im Weltraumvertragssystem und im Seerechtsübereinkommen formuliert und wirksam wurden, waren sämtlich territorial begründet - sie basierten auf den vorherrschenden Prinzipien der territorialen Ressourcenaufteilung und sie implementierten diese Prinzipien. In diesem Abschnitt sollen die Commons als eine neue Qualifizierung des Territorialitätsprinzips untersucht werden. Der Diskurs der Commons, so die These, betrieb Souveränitätspolitik mit anderen Mitteln.

45 Marcus Haward/Tom Griffiths (Hg.): Australia and the Antarctic Treaty System. 50 Years of Influence. Sydney u.a. 2011; Peder Roberts: The European Antarctic. Science and Strategy in Scandinavia and the British Empire. New York 2011. 
Die enge Verflechtung von Souveränität und Territorialität kommt in der problematischen Vorwegnahme zum Ausdruck, nach der jedes Commons-System notwendig an den Vorgaben der Endlichkeit und Freiwilligkeit scheitere - der ultimativen Endlichkeit der Ressourcen sowie der Unmöglichkeit in der Zurückhaltung ihrer Konsumenten. Hardin versah die völkerrechtlichen Debatten mit einer anthropologischen Begründung dafür, dass eine Gemeinwirtschaft auch unter den wohlmeinendsten philosophischen Prinzipien fehlschlagen musste. So war er der Ansicht, dass Jeremy Benthams Regel des Utilitarismus, stets so $\mathrm{zu}$ handeln, dass „the greatest good for the greatest number“ produziert werde, unmöglich zu realisieren sei. Dazu berief er sich auf die absoluten Grenzen der Erde. Spätestens seit den spektakulären Bildern des Blauen Planeten, aufgenommen von den Apollo-Besatzungen auf dem Weg zum Mond, sei der Eindruck der absoluten Begrenztheit irdischer Ressourcen zur Wirklichkeit geworden. Seiner Ansicht nach konnte ein Commons-System nur in einer unendlichen Welt, in der die ökologische Tragfähigkeit noch längst nicht ausgeschöpft sei, erfolgreich sein.

But it cannot work in a world that is reaching its limits, in which the decisions being made overstress the carrying capacity of the environment. ${ }^{46}$

Neben der endlichen Tragfähigkeit problematisierte Hardin das „System der freiwilligen Zurückhaltung“, das in einem Commons-System den Nutzern einer Ressource nahegelegt würde. ${ }^{47}$ Es könne jederzeit durch den einzelnen Akteur korrumpiert werden, der sich der Selbstbeschränkung verweigere. Appelle an das biblische Gebot des „Thou shalt not“ oder „Du sollst nicht“ allein seien nicht ausreichend. Das traditionelle Gesetz sei stattdessen durch administrative Gesetzgebung und eine machtvolle Exekutive durchzusetzen. Dazu sei es notwendig, „corrective feedbacks“ in Form von gesetzlichen Zwängen in gesellschaftliche Prozesse einzubeziehen, die kollektiv oder zumindest mehrheitlich befürwortet würden. ${ }^{48}$ Die kollektive Ressourcennutzung sollte auf vereinbarte und institutionalisierte Formen der Nötigung aufsetzen, die Hardin umschreibt als „the necessity of coercion for all - mutual coercion, mutually agreed upon“. ${ }^{49}$

46 Hardin, Exploring New Ethics for Survival (1972), 118.

47 Ebd., 117: „system of voluntary restraint“.

48 Hardin, The Tragedy of the Commons (1968), 1246.

49 Hardin, Exploring New Ethics for Survival (1972), 130, Hervorhebung im Original. 
Frei nach Hegel, so Hardin, verträte er die Ansicht, dass Freiheit die Anerkennung der Notwendigkeit sei: „Freedom is the recognition of necessity. “50 Wieder führt er das Beispiel der Weltmeere an. Alternativ zu deren Privatisierung durch fiktives Einzäunen wäre deren Sozialisierung denkbar, doch dazu sei zunächst eine durchschlagkräftige internationale Instanz zu schaffen:

Socialize the oceans: create an international agency with teeth. ${ }^{51}$

Diese Instanz sollte „Verantwortung“ und „Gewissen“ rechtsverbindlich einfordern, Eigenschaften, die es Hardin zufolge in der modernen Welt nach Darwin und Freud nicht mehr geben konnte.52 Doch existierte keine Institution, die terrestrische und extraterrestrische Räume wie die Tiefsee, die Antarktis und den Weltraum hätte vertreten und außerhalb der tradierten Rechtsformen verhandeln können. Der Völkerbund und die Vereinten Nationen hielten zwar das Mandat, sich als vermittelnde und entscheidende Instanzen in internationalen Angelegenheiten zu positionieren und Eigentums- und Nutzungsfragen verbindlich und möglichst gerecht zu klären. ${ }^{53}$ Doch stellten sie im strengen Sinne keine supranationalen Organisationen dar. Ihre Vertragssysteme wurden auf nationalstaatlicher Grundlage und durch die Nationalstaaten geschlossen. Die Staaten waren die als legitime Vertragspartner anerkannten Akteure, wenn es um international bindende Vertragswerke ging. Die exekutiven Möglichkeiten der internationalen Staatenorganisationen schienen jedoch begrenzt. Es war dieses Problem, die Frage des Quis custodiet ipsos custodies? (Wer bewacht die Wächter?), die Hardin als eine der wichtigsten politischen Fragen seiner Zeit betrachtete. ${ }^{54}$

Im Jahre 1957 beschrieb der deutsch-amerikanische Politikwissenschaftler John H. Herz seine Beobachtung, dass souveräne Staaten ihre gemeinsamen Interessen gegenseitig zunehmend in der Form extensiver Kooperationen anerkannten, während sie zugleich ihre jeweilige Unabhängigkeit respektierten und geltend machten. Herz formulierte die These vom Aufstieg und Niedergang des

50 Ebd., 132. Hardin zitierte hier genau genommen Friedrich Engels’ Referenz an Hegel, für welchen „Freiheit die Einsicht in die Notwendigkeit“ gewesen sei.

51 Ebd., 121, Hervorhebung im Original.

52 Hardin, The Tragedy of the Commons (1968), 1246f. (Zitate im Original: „conscience“, „,responsibility“).

53 Iris Schröder/Susanne Schattenberg/Jan-Holger Kirsch (Hg.): Internationale Ordnungen und neue Universalismen im 20. Jahrhundert, in: Zeithistorische Forschungen/Studies in Contemporary History, Online-Ausgabe, 8 (2011), 3. http://www.zeithistorische-forschungen.de/16126041Inhalt-3-2011 (17. März 2014).

54 Hardin, Exploring New Ethics for Survival (1972), 134. 
Territorialstaates, der in seiner Einheit, Kompaktheit und Kohärenz durch das Prinzip der „Territorialität“ charakterisiert sei, durch den identifizierbaren abgegrenzten und zu schützenden zweidimensionalen Raum. ${ }^{55}$ Mit der Verbindung realistischer und idealistischer Perspektiven des politischen Handelns machte Herz sich in den Politikwissenschaften der internationalen Beziehungen und in der Völkerrechtslehre einen Namen. Er erweiterte gleichsam Hardins Naturrechtslehre und seine spieltheoretischen Ansätze rationalen Handelns um eine soziale Perspektive, die internationale politische Systeme als nicht-rationale Systeme theoretisierte. Herz gilt als Begründer des „Sicherheitsdilemmas“, das die nötige Verhandlungs- und Vermittlungsarbeit in Situationen thematisierte, die nur vermeintlich durch rationale Interessen und rationales Handeln bestimmt waren.

Herz begründete den Niedergang des souveränen territorialen Staates sicherheitsstrategisch und ausschließlich militärisch: Mit dem Schwinden der Möglichkeiten territorialer Verteidigung sei die Basis territorialer Macht weggebrochen. Dies sei der neuen Permeabilität des Atomzeitalters geschuldet, der vertikalen Macht der Zerstörung aus der Luft und aus dem All, die sich über den zweidimensionalen Raum hinwegsetzte und die noch so große Stärke eines Staates mit seiner größtmöglichen Vulnerabilität zusammenfallen ließ. Während das nationalstaatliche System eine relativ friedliche und bemerkenswert stabile Koexistenz der Nationen ermöglicht habe, die unter dem Motto der balance of power ausgehandelt worden sei, aber immer wieder neu verstetigt werden müsse, sei das gegenwärtige Zeitalter nicht länger durch impermeable Grenzen, sondern durch ein absolutes Ausgesetztsein gekennzeichnet. Statt zwischenstaatlicher Grenzziehungen und imperialer Verschiebungen von Grenzen erfahre die Welt nun das Ende der world frontier und die Auswirkungen einer prinzipiell geschlossenen Erde. Herz führte die Nachfrage nach einem neuen System der kollektiven Sicherheit, das den Nationalstaaten auch und vor allem in Zeiten endlicher Ressourcen Sicherheit gewährleiste, auf diese Entwicklung zurück, in der die alte Expansionspolitik des Westens zu ihrem Ende kam. ${ }^{56}$

55 „As a matter of fact, the meaning and function of the basic protective unit, the ,sovereign“ nation-state itself, have become doubtful. On what, then, can policy and planning be built?", in: John H. Herz: Rise and Demise of the Territorial State, in: World Politics 9 (1957), 4, 473-493, hier 473.

56 Herz, Rise and Demise (1957), 474f., 481f. Zum Ende der world frontier siehe das Buch des Historikers Walter Prescott Webb: The Great Frontier. Boston 1952. Der Historiker Charles S. Maier schlug mit Bezug auf Herz vor, „Territorialität“als das strukturierende Prinzip des Jahrhunderts zwischen 1860 und 1970 anzuerkennen: Charles S. Maier: Consigning the Twentieth Century to History. Alternative Narratives for the Modern Era, in: American Historical Review 105 (2000), 807-831. 
Die Beobachtungen des Politikwissenschaftlers, die Menschen seien „Einwohner eines Planeten von begrenzter (und, wie es heute scheint, ungenügender) Größe“, lassen sich ähnlich im zeitgenössischen historischen, ökologischen und ökonomischen Diskursen über die neuen Grenzen der Erde hinsichtlich der globalen Ressourcenökonomie ausmachen. ${ }^{57}$ Herz selbst vollzieht diese Erweiterung vom „common interest of all mankind in sheer survival“ aufgrund der konstanten Bedrohung nuklearer Auslöschung hin zu universellen Interessen in Weltangelegenheiten wie dem Dilemma von Bevölkerungswachstum, Ressourcenerschöpfung und Umweltvergiftung. ${ }^{58}$ Doch seien die neu geschaffenen internationalen Systeme trotz des zu beobachtenden Anstiegs multilateraler Organisationen und Regelwerke nicht innovativ, sondern konservativ - sie seien darauf angelegt, die territoriale Integrität und politische Unabhängigkeit der Staaten zu garantieren. ${ }^{59}$

Gut zehn Jahre später, im Jahr 1968, spitzte Herz den beschriebenen Wandlungsprozess weiter zu. Er behauptete, dass es nicht das ganz Andere sei, das in der Form des globalen Universalismus und einer Menschheit, die sich selbst als Einheit verstehe, den Platz der Territorialität einnehme, sondern eine Politik des retrenchment, der Wiederverschanzung, einer erneuten Begrenzung und $\mathrm{Zu}$ schneidung von Raum, und eine Tendenz ,toward area not losing its impact but regaining it; in short, trends toward a ,new territoriality “ “. ${ }^{60}$ Herz motivierte sein Konzept der neo-territoriaity, der neu entstandenen Multipolarität der Welt, die in alten territorialen Mustern verblieb, wieder sicherheitsstrategisch. Doch lässt sich seine Beobachtung auf zeitgenössische Aushandlungen um die Allokation (globaler) Güter erweitern. Die Debatte um das Gemeinsame Erbe der Menschheit

57 Herz, Rise and Demise (1957), 489 (Zitat im Original: „we are inhabitants of a planet of limited (and, as it now seems, insufficient) size“). Kenneth E. Boulding: The Economics of the Coming Spaceship Earth, in: Henry Jarrett (Hg.): Environmental Quality in a Growing Economy. Essays from the Sixth RFF Forum on Environmental Quality held in Washington, March 8 and 9, 1966. Baltimore 1966, 3-14.

58 Herz, Rise and Demise (1957), 492.

59 Ebd., 484.

60 Zitat im Original: „The theory of ,classical territoriality“ and of the factors of threatening its survival stands. But I am no longer sure that something very different is about to take its place. There are indicators pointing in another direction: not to ,universalism' but to retrenchment; not to interdependence but to a new self-sufficiency; toward area not losing its impact but regaining it; in short, trends toward a ,new territoriality“. John H. Herz: The Territorial State Revisited: Reflections on the Future of the Nation-State, in: Polity 1 (1968), 1, 11-34, hier 13. Herz argumentiert den Trend hin zu Multipolarität erneut sicherheitsstrategisch: Die nationale Sicherung geschehe durch die Proliferation von Wissen, Information und Knowhow über atomare Rüstungstechnik, so dass stabilisierende Systeme gegenseitiger Abschreckung entstünden. 
verdeutlichte die Spannung: Die neuen Instanzen und Programme der multilateralen und kooperativen globalen Gestaltung beruhten nicht mehr ausschließlich auf den traditionellen Territorialisierungsstrategien, ebenso wenig aber konnten sich neue Ideen der Kollektivierung effektiv durchsetzen.

Internationalisierungsstrategien, die wie das Prinzip des Common Heritage of Mankind auf die Endlichkeit der globalen Ressourcen, auf die Verantwortlichkeiten und auf die Verbindlichkeit vereinbarter Nutzungsformen hinwiesen, stellten demnach, will man Herz folgen, ein Ergebnis eines erweiterten zeitgenössischen Sicherheitsdilemmas dar. Ein neues Umweltsicherheitsdilemma - im weitesten Sinne des anthropozentrischen Welt- und Umweltgedankens - ging mit einer neuen Form der Territorialpolitik unter den Bedingungen der globalen Unsicherheit und Interdependenz einher. Die neue Territorialpolitik war Ausdruck der fortgesetzten Geltung nationaler Interessen und der Anerkennung der Nationalstaaten als einzig legitime Verhandlungspartner in einer Welt multipolarer, eigenständiger territorialer Einheiten, die in wechselnden Bündnissen und Zusammenschlüssen zunehmend globale Anliegen und Ideale verfolgten. ${ }^{61}$

\section{Zusammenfassung: Commoning und Uncommoning}

Die Jahre um 1970 waren zentral in der Entstehung eines Denkens über und eines Umgangs mit irdischem und außerirdischem Raum in Begriffen der globalen Gemeinschaftsgüter. Insbesondere neu erschlossene Räume wie die Tiefsee, die Polargebiete und das Weltall wurden zu Gegenständen internationaler Organisationen und Verhandlungen sowie unter dem CHM-Prinzip verhandelt. Doch markierten die Verhandlungen nicht nur einen Wandel hin zu neuen Praktiken des Commoning, des aktiven Erhalts der Commons mit Bezug auf die Nutzergemeinschaft oder ",community“, ${ }^{62}$ sondern sie verdeutlichten auch die Tendenz des Uncommoning von terrestrischem und extraterrestrischem Raum durch gegenläufige Abgrenzungs- und Ausgleichsprozesse. Der moralische Imperativ, der mit der Rede von den gemeinsamen Interessen, Zukünften und Verantwortlich-

61 Ebd., 31 („security dilemma“).

62 „Commoning“ bezeichnet die Praxis des Erhalts der Commons und bezieht sich auf die Nutzergemeinschaft oder „community“. Siehe Silke Helfrich/Heinrich Böll Stiftung (Hg.): Commons. Für eine neue Politikjenseits von Markt und Staat. Bielefeld 2012, 19 und Kapitel III: Commoning Soziale Innovationen weltweit, $263 \mathrm{ff}$. 
keiten der Menschheit nahegelegt wurde, blendete die unterschiedlichen Positionen der betreffenden Gebiete im Weltgefüge aus, aber er hob sie nicht auf. Dieser Beitrag argumentiert, dass die entstehenden Commons-Regime die Spannungen zwischen traditionellen territorial basierten und gemeinschaftlichen Eigentumsund Souveränitätsprinzipien nicht auflösten, sondern auf die Ebene einer neuen internationalen Gestaltung der Welt transferierten.

Seither wurde viel mit den Commons experimentiert. Einerseits beobachten wir, mit der Kultur- und Politikwissenschaftlerin Kathryn Milun gesprochen, die immer stärkere Auflösung der Commons in die political uncommons, die vielfältigen, lokalen und fragmentierten Weisen der politischen Aneignung von Raum. ${ }^{63}$ Nichtregierungsorganisationen wie Greenpeace nehmen jetzt selbstverständlich für sich in Anspruch, die Politik des Commoning voranzutreiben, um nationalstaatliche Territorialität auszuhebeln, wie in den jüngeren Kontroversen um die Polargebiete zu beobachten ist. In den 1980er Jahren erklärte Greenpeace die Antarktis zum „Weltpark“, um die Ausbeutung mineralischer Rohstoffe zu verhindern. In einseitiger Verfügung stellte die Organisation dem CHM-Prinzip das Schutzregime des Parks entgegen, um die antarktischen Ressourcen gänzlich aus der Vorstellung der allgemeinen Verfügbarkeit herauszunehmen. ${ }^{64}$ Andererseits entstehen neue Commons-Konzepte, die Ressourcen und ihre Allokation neu $\mathrm{zu}$ fassen und $\mathrm{zu}$ verhandeln suchen. Zum einen sind es die common pool resources oder common property resources, die nach Zugangsmöglichkeiten und nach Strittigkeit einer Ressource differenzieren und so ein deutlich komplexeres Bild möglicher Nutzungskonflikte zeichnen können. ${ }^{65}$ Zum anderen sind es neue sozial- und kulturwissenschaftliche Commons-Konzepte, die selbst auf die vermeintlich evidente Vorstellung von Knappheit verzichten wollen, die allen Debatten um die globalen Gemeinschaftsgüter um 1970 zugrunde gelegt wurde. In expliziter Kritik an Hardins Thesen machen sie eine Vorstellung der „Generativen Commons“ geltend, die nicht auf Knappheit, sondern auf schöpferische natürliche Fülle setzt. ${ }^{66}$ Damit wurde auch die womöglich letzte vermeintliche Selbstverständlichkeit über natürliche Ressourcen und globale Güter in konstruktivistischer Absicht hinterfragt. Die neuen Commons seien produktiv, ohne

63 Kathryn Milun: The Political Uncommons. The Cross-Cultural Logic of the Global Commons. Burlington 2011.

64 Baslar, The Concept of the Common Heritage of Mankind in International Law (1998), $258 \mathrm{ff}$.

65 Dan Brockington/Rosaleen Duffy/Jim Igoe: Nature Unbound. Conservation, Capitalism and the Future of Protected Areas. London 2008.

66 Silke Helfrich/David Bollier: Commons als transformative Kraft, in: Silke Helfrich/Heinrich Böll Stiftung (Hg.): Commons. Für eine neue Politik jenseits von Markt und Staat. Bielefeld 2012, 15-23, hier 19. 
in erster Linie für den Markt zu produzieren. Die Commons seien konstruktiv und sie seien selbstverständlich konstruiert, denn sie existierten für und durch die Menschen, niemals ohne sie.

Die Verfechter dieser neuen Commons-Begriffe setzen auf ökonomische Theorien der Selbstorganisation. Der Ökonomin und Nobelpreisträgerin Elinor Ostrom zufolge können Commoning-Strategien gelingen, wenn Entscheidungen und Regeln über gemeinschaftlich genutzte Güter durch jene getroffen und angepasst würden, die von einem Gemeinschaftsgut auf die eine oder andere Weise berührt seien - wobei schon diese Zuordnung kritisch sein dürfte, denn sie lässt Fragen der technisch-wissenschaftlichen Innovation, der Konstitution von und des $\mathrm{Zu}$ gangs zu Ressourcen, ob natürlich oder kulturell, außer Acht. ${ }^{67}$ Die internationalen Auseinandersetzungen über Räume wie die Tiefsee, die Polgebiete und vor allem das Weltall, die bis weit in das 20. Jahrhundert hinein als praktisch ohne Belang für das Menschengeschehen galten und als ,Gemeinschaftsgüter' erst mit ihrer Ressourcenhaftigkeit zur Existenz kamen, zeigen, dass weder soziale, kulturelle und historische Traditionen noch wissenschaftlich-technische Möglichkeiten der Konstruktion von Natur als Ressource unterschätzt werden sollten, will das Commoning nicht eine preisgekrönte Theorie bleiben.

67 Elinor Ostrom: Governing the Commons. The Evolution of Institutions for Collective Action. Cambridge 1990 . 